\title{
Подавление температурной зависимости длины волны излучения в светодиодных структурах со ступенчатым гетеропереходом II типа InAsSb/InAsSbP
}

\author{
(C) А.А. Семакова ${ }^{1}$, В.В. Романов ${ }^{2}$, Н.Л. Баженов ${ }^{2}$, К.Д. Мынбаев ${ }^{2}$, К.Д. Моисеев ${ }^{2}$ \\ ${ }^{1}$ Университет ИТМО, \\ 197101 Санкт-Петербург, Россия \\ ${ }^{2}$ Физико-технический институт им. А.Ф. Иоффре Российской академии наук, \\ 194021 Санкт-Петербург, Россия \\ E-mail: antonina.semakova@itmo.ru
}

Поступила в Редакцию 6 ноября 2020 г.

В окончательной редакции 16 ноября 2020 г.

Принята к публикации 16 ноября 2020 г.

Представлены результаты исследования электролюминесценции асимметричных светодиодных гетероструктур InAs/InAs ${ }_{1-y} \mathrm{Sb}_{y} / \mathrm{InAsSbP}$ с мольной долей InSb в тройном твердом растворе активной области $y=0.15$ и $y=0.16$ в диапазоне температур $4.2-300 \mathrm{~K}$. По данным экспериментов определено формирование ступенчатого гетероперехода II типа на гетерогранице $\mathrm{InAs}_{1-y} \mathrm{Sb}_{y} / \mathrm{InAs}_{0.41} \mathrm{Sb}_{0.28} \mathrm{P}_{0.40}$. Показан доминирующий вклад интерфейсных излучательных переходов на гетерогранице II типа в диапазоне температур $4.2-180 \mathrm{~K}$, позволяющий минимизировать температурную зависимость рабочей длины волны излучения светодиода.

Ключевые слова: гетеропереходы, арсенид индия, антимониды, электролюминесценция.

DOI: 10.21883/FTP.2021.03.50607.9549

\section{1. Введение}

В настоящее время ведется интенсивная разработка сенсоров, работающих в средней инфракрасной области спектра (2-6 мкм) и предназначенных для экологического мониторинга, медицинской диагностики и прецизионного контроля технологических процессов в различных отраслях промышленности $[1,2]$. Перспективными источниками излучения для таких устройств являются светодиоды на основе гетероструктур с активной областью на основе твердых растворов InAsSb [2]. Необходимость в таких источниках излучения достаточно велика, в частности, они требуются для анализа состава атмосферы и определения концентрации таких химических соединений, как, например, оксид азота (полоса поглощения вблизи 5.3 мкм) [3].

Разработка длинноволновых $(\lambda>5$ мкм $)$ источников излучения на основе данных материалов затруднена тем, что увеличение содержания атомов сурьмы в эпитаксиальном слое InAsSb ведет к рассогласованию по параметру кристаллической решетки наращиваемого тройного твердого раствора относительно соответствующего параметра подложки InAs. При создании узкозонных гетероструктур методом жидкофазной эпитаксии (ЖФЭ) проблема рассогласования параметров решетки решалась формированием метаморфных буферных слоев [4,5]. В то же время использование метода газофазной эпитаксии из металлоорганических соединений (МОГФЭ) позволяло получать гетероструктуры с высоким содержанием InSb в активной области InAsSb непосредственно на подложке InAs [6-8]. Еще одним преимуществом МОГФЭ является возможность наращивания четверного твердого раствора InAsSbP, изопериодного к InAs, во всем диапазоне составов, что позволяет формировать потенциальные барьеры как для электронов, так и для дырок, и тем самым значительно улучшать локализацию носителей заряда в окрестности узкозонной активной области [8].

Настоящая работа является развитием исследований электролюминесценции (ЭЛ) асимметричных светодиодных гетероструктур $n$-InAs/ $n-\mathrm{InAs}_{1-y} \mathrm{Sb}_{y} / p$-InAsSbP с мольной долей $\mathrm{InSb}$ в твердом растворе активной области до $y=0.16[9,10]$. В работе представлены новые результаты по изучению особенностей температурных зависимостей спектральных характеристик данных структур.

\section{2. Методика экспериментов}

Гетероструктуры были получены методом МОГФЭ на нелегированных подложках $\operatorname{InAs}(001)$ с концентрацией электронов, обусловленной наличием остаточных примесей, $n=3 \cdot 10^{16} \mathrm{~cm}^{-3}(T=300 \mathrm{~K})$. Эпитаксиальное осаждение слоев твердых растворов InAsSb и InAsSbP проводилось в горизонтальном реакторе с резистивным типом нагрева при атмосферном давлении. Детали технологии получения гетероструктур были представлены ранее в работе [10]. Для проведения сравнительного анализа были выбраны две гетероструктуры, различающиеся составом активной области: InAs/InAs ${ }_{0.85} \mathrm{Sb}_{0.15} / \mathrm{InAs}_{0.41} \mathrm{Sb}_{0.28} \mathrm{P}_{0.40}$ (образец 317) и $\operatorname{InAs} / \mathrm{InAs}_{0.84} \mathrm{Sb}_{0.16} / \mathrm{InAs}_{0.41} \mathrm{Sb}_{0.28} \mathrm{P}_{0.40}$ (образец 315 ). Толщина активной области InAsSb составляла 3 мкм, а широкозонного барьерного слоя InAsSbP - 1.2 мкм в обеих структурах. Материал активной области не леги- 
ровался. В качестве легирующей акцепторной примеси для барьерного слоя применялся цинк.

Светодиодные чипы были выполнены в виде мезаструктур размером $400 \times 400$ мкм. Чипы монтировались на корпуса типа ТО-18. Спектры ЭЛ регистрировались в диапазоне температур $T=4.2-300 \mathrm{~K}$ при импульсном возбуждении с частотой 1 кГц и длительностью импульса 2 мкс. В качестве детектора использовались фотодиод $\mathrm{InSb}$ и фоторезистор $\mathrm{HgCdTe}$.

\section{3. Результаты и обсуждение}

На рис. 1 представлены спектры ЭЛ исследуемых гетероструктур, полученные при $T=4.2 \mathrm{~K}$. Видно, что данные спектры содержат две выраженные полосы излучения: первая - доминирующая по интенсивности и достаточно узкая с максимумом излучения вблизи 3.11 мкм, и вторая - менее интенсивная и более широкая, в диапазоне длин волн 3.5-5.5 мкм. Ширина на половине высоты интенсивности („полуширина“) составила $\sim 10$ мэВ для коротковолновых полос излучения и 50-60 мэВ для длинноволновых полос. Глубокий провал в спектрах ЭЛ вблизи $\lambda=4.3$ мкм обусловлен поглощением выходящего излучения молекулами углекислого газа $\left(\mathrm{CO}_{2}\right)$, присутствующего в атмосфере.

Основываясь на данных по энергетическому положению спектрального максимума коротковолновых полос излучения и их полуширине, можно предположить, что наличие упомянутых полос ЭЛ связано с рекомбинацией носителей заряда в объеме подложки InAs. В исследуемых гетероструктурах $n$-InAs $/ n-\mathrm{InAs}_{1-y} \mathrm{Sb}_{y} / p$-InAsSbP коротковолновая полоса ЭЛ рассматривается как дополнительный канал излучательной рекомбинации и является фактором, изучение которого может позволить определить природу утечки инжектируемых носителей заряда из активной области структуры. Для проверки нашего предположения было проведено исследование температурной зависимости ЭЛ одиночной гетероструктуры $n^{0}$-InAs $/ n^{0}-\mathrm{InAs}_{0.32} \mathrm{Sb}_{0.26} \mathrm{P}_{0.42}$, полученной путем наращивания твердого раствора InAsSbP на подложку, которая применялась при создании исследуемых структур 315 и 317. Толщина слоя InAsSbP в одиночной гетероструктуре составляла 0.6 мкм. В силу особенностей зонной структуры (наличия существенного электронного ограничения в InAs), основной сигнал ЭЛ данной гетероструктуры определялся излучательной рекомбинацией носителей заряда в подложке: полученные спектры содержали четко выраженную полосу излучения в диапазоне длин волн 3-3.2 мкм, аналогично подобной одиночной гетероструктуре, полученной другим технологическим способом (ЖФЭ) [11].

На рис. 2, $a$ представлены спектры ЭЛ одиночной гетероструктуры $\quad n^{(0)}$-InAs $/ n^{(0)}-\mathrm{InAs}_{0.32} \mathrm{Sb}_{0.26} \mathrm{P}_{0.42}$, зарегистрированные при температурах $T=4.2$ и 63 К. Как видно из верхнего графика, спектр ЭЛ с максимумом при 0.40 эВ и полушириной 11 мэВ при

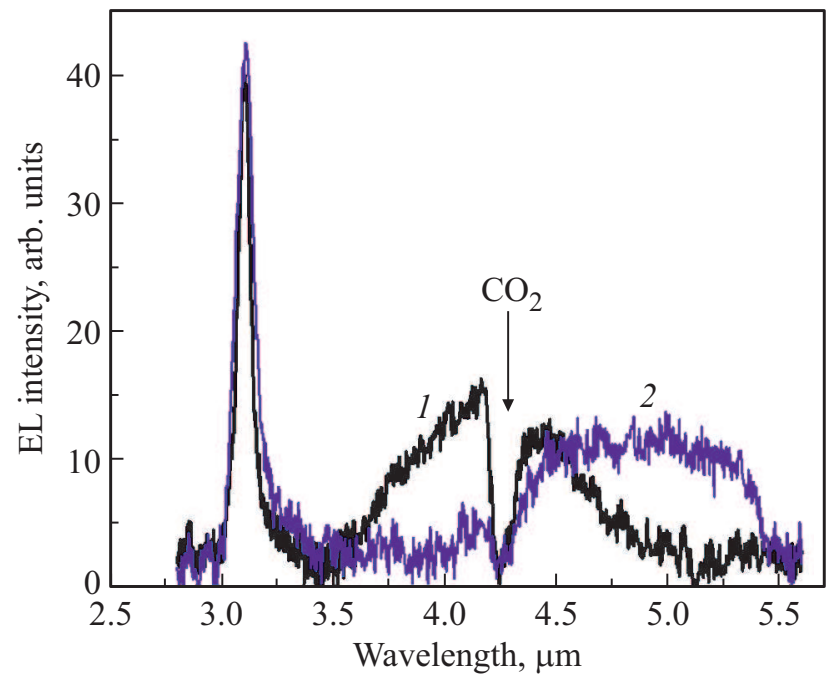

Рис. 1. Спектры ЭЛ гетероструктур 317 (спектр 1) и 315 (спектр 2) при $T=4.2 \mathrm{~K}$ и токах накачки $i=1 \mathrm{~A} \mathrm{(317)} \mathrm{и}$ $i=3 \mathrm{~A}(315)$.

низких температурах имел асимметричную форму за счет появления высокоэнергетического плеча вблизи 0.409 эВ. При повышении температуры $(T>60 \mathrm{~K})$ проявлялась дуплексная структура спектра ЭЛ, при которой основная полоса излучения состояла из двух четко выраженных полос (рис. 2, $a$, нижний график). При этом общая полуширина полосы ЭЛ увеличилась до 31 мэВ и спектр сместился в сторону меньших энергий. С дальнейшим увеличением температуры высокоэнергетическая полоса излучения становилась доминирующей, и в диапазоне температур $148<T<300 \mathrm{~K}$ в спектре ЭЛ оставалась одна полоса излучения в области энергий 0.389-0.357 эВ. В спектрах ЭЛ гетероструктур 317 и 315 наблюдалась только низкоэнергетическая полоса излучательных рекомбинационных переходов в InAs. На рис. 2, $b$ представлена температурная зависимость спектрального положения максимума данной полосы излучения для гетероструктуры 315 . Поскольку наличие высокоэнергетических полос в спектрах ЭЛ определяется излучательной рекомбинацией в подложке InAs, температурная зависимость ширины запрещенной зоны $E_{g}(T)$ должна описываться линейно-квадратичным соотношением, предложенным Варшни [12]:

$$
E_{g}=E_{0}-\alpha \cdot T^{2} \cdot(T+\beta)^{-1},
$$

где $E_{0}=0.418$ эВ ( $E_{g}$ при $T=0 \mathrm{~K}$ для $\left.\mathrm{InAs}\right), \alpha$ и $\beta$ - константы $\left(\alpha=2.76 \cdot 10^{-4}\right.$ эВ/K, $\beta=93 \mathrm{~K}$ для InAs) [13].

Из рис. 2, $b$ видно, что максимумы высокоэнергетических полос ЭЛ гетероструктуры $n^{0}$-InAs/ $n^{0}-$ InAs $_{0.32} \mathrm{Sb}_{0.26} \mathrm{P}_{0.42}$ и экспериментальные данные, полученные ранее из спектров фотолюминесценции (ФЛ) подложки InAs [9], хорошо согласуются с расчетной зависимостью $E_{g}(T)$ для арсенида индия во всем диапазоне температур. Следовательно, данные излучательные 

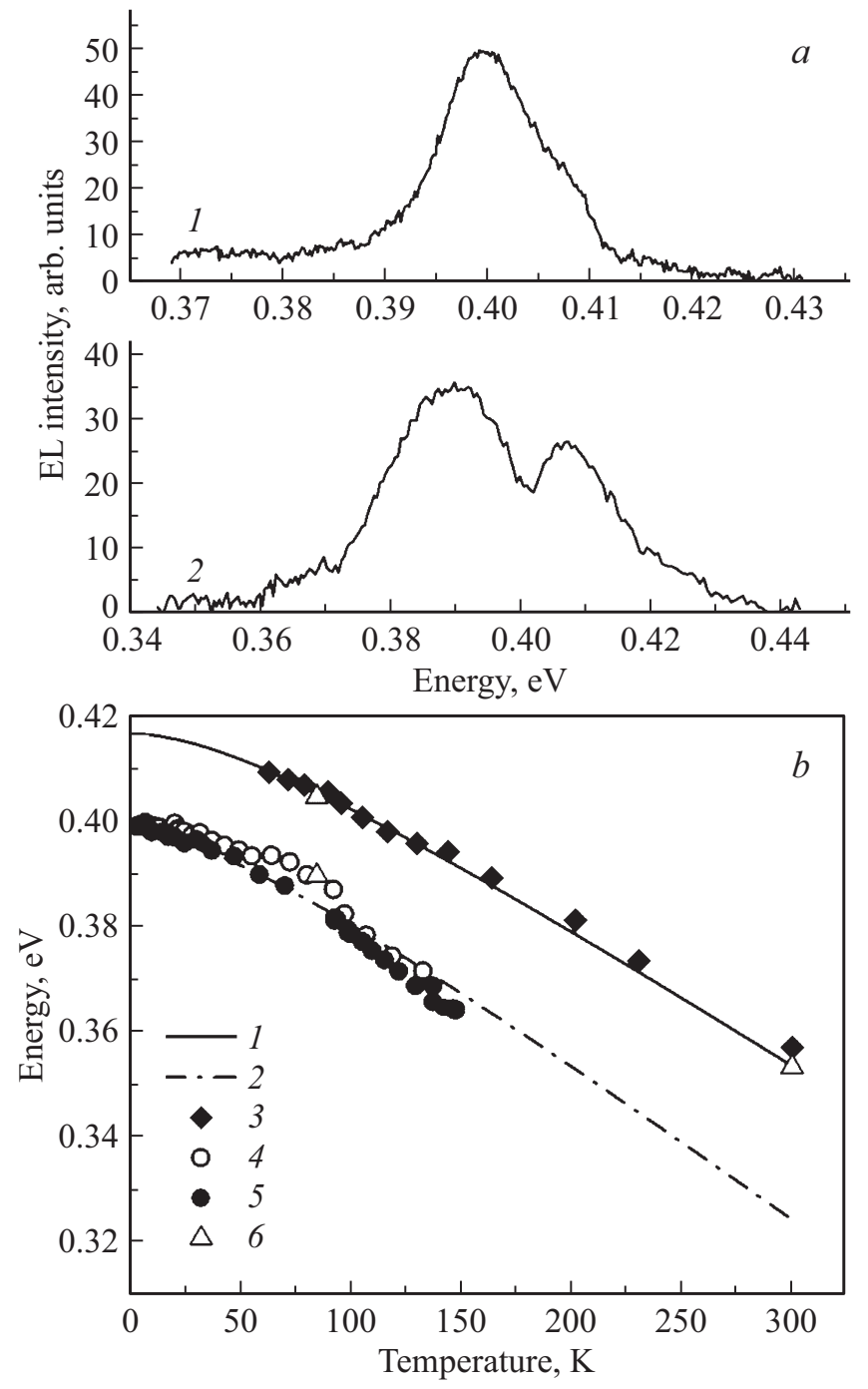

Рис. 2. Спектры ЭЛ гетероструктуры $n^{0}$-InAs/ $n^{0}-\operatorname{InAs}_{0.32} \mathrm{Sb}_{0.26} \mathrm{P}_{0.42}$ при токе накачки $i=1 \mathrm{~A}$ и температурах $4.2 \mathrm{~K}$ (спектр 1) и $63 \mathrm{~K}$ (спектр 2) (a), и температурная зависимость ширины запрещенной зоны InAs, рассчитанная по выражению (1) (кривая 1); экспериментальные данные ЭЛ гетероструктуры $n^{0}$-InAs $/ n^{0}-\operatorname{InAs}_{0.32} \mathrm{Sb}_{0.26} \mathrm{P}_{0.42}$ : максимумы высокоэнергетической (символы 3) и низкоэнергетической (символы 5) полос излучения; данные из спектров ФЛ подложки InAs [9] (символы 6); аппроксимация согласно соотношению (1) с параметрами $\alpha=3.1 \cdot 10^{-4}$ эВ/K, $\beta=80 \mathrm{~K}$ (кривая 2); данные ЭЛ гетероструктуры 315 (символы 4 ) (b).

переходы можно отнести к межзонной рекомбинации в объеме InAs. Вторая часть экспериментальных данных (низкоэнергетическая часть полос ЭЛ структуры $n^{0}$-InAs $/ n^{0}-\mathrm{InAs}_{0.32} \mathrm{Sb}_{0.26} \mathrm{P}_{0.42}$ и высокоэнергетические полосы в спектрах ЭЛ гетероструктур 315 и 317) также демонстрирует хорошее соответствие температурных зависимостей. Полученные результаты с хорошей точностью аппроксимируются зависимостью Варшни с параметрами $\alpha=3.1 \cdot 10^{-4}$ эВ/K и $\beta=80 \mathrm{~K}$, близкими к соответствующим значениям для InAs. При этом при построении данной кривой за величину $E_{0}$ было принято значение 0.398 эВ, характерное для энергетических переходов, типичных для рекомбинации донорноакцепторных пар в нелегированном InAs [14]. Таким образом, можно сделать вывод, что высокоэнергетические полосы, наблюдаемые в спектрах ЭЛ гетероструктур 315 и 317 в диапазоне температур 4.2-150 K, были вызваны рекомбинацией с участием донорно-акцепторных пар, обусловленных остаточными примесями и дефектами в подложке InAs.

На рис. 3 представлены температурные зависимости спектрального положения максимумов длинноволновых полос излучения гетероструктур 315 и 317, а также приведены расчетные зависимости $E_{g}(T)$ согласно соотношению (1) для двух составов тройного твердого раствора $\operatorname{InAs}_{1-y} \mathrm{Sb}_{y}: y=0.15$ (образец 317) и $y=0.16$ (образец 315). Значения $E_{0}$ для твердых растворов InAsSb вычислялись как интерполяция долей бинарных соединений InAs и InSb согласно выражению, предложенному в работе [15]:

$$
E_{g}=E_{\mathrm{InAs}} \cdot(1-y)+E_{\mathrm{InSb}} \cdot y-C_{\mathrm{InAsSb}} \cdot y \cdot(1-y),
$$

где $\quad E_{\mathrm{InAs}}=0.418$ эВ,$\quad E_{\mathrm{InSb}}=0.235$ эВ $\quad$ и $\quad C_{\mathrm{InAsSb}}=$ $=0.61$ эВ [16]. Коэффициенты $\alpha$ и $\beta$ принимались равными соответствующим параметрам для InAs. Из рис. 3 видно, что хорошее согласие экспериментальных данных и расчетных кривых наблюдается при высоких температурах $(T>180 \mathrm{~K})$; это указывает на межзонный характер излучательной рекомбинации в узкозонной области данных гетероструктур при этих температурах. Кроме того, следует отметить, что в данном интервале температур исследуемые образцы воспроизводили температурную зависимость $E_{g}(T)$ матрицы, в качестве которой выступала подложка InAs.

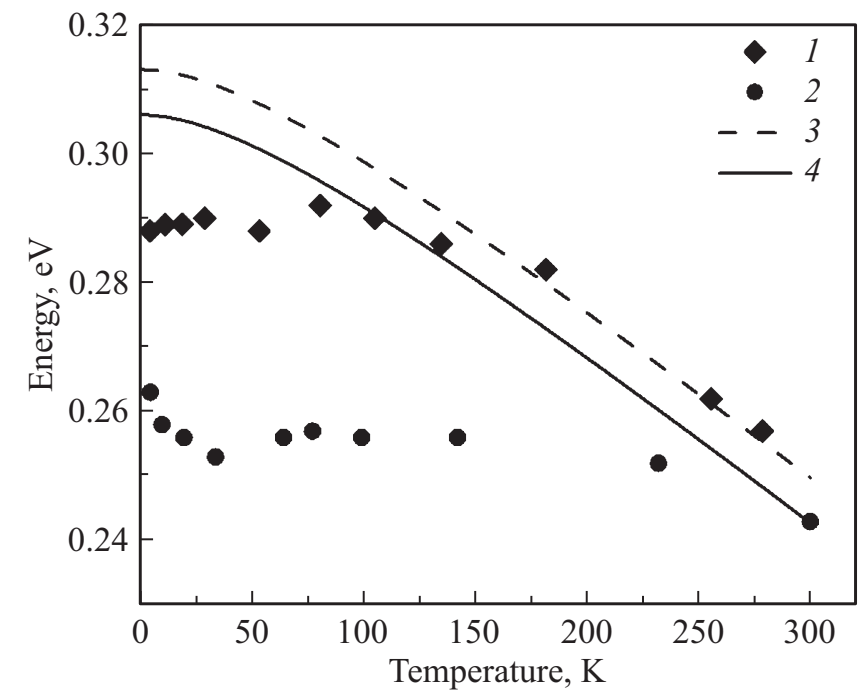

Рис. 3. Расчетные температурные зависимости ширины запрещенной зоны активной области InAsSb гетероструктур 317 (кривая 3) и 315 (кривая 4) и экспериментальные значения энергии фотона в максимуме низкоэнергетической полосы ЭЛ для гетероструктур 317 (символы 1) и 315 (символы 2). 
В области низких температур $(T<150 \mathrm{~K})$ наблюдалась существенная разница между расчетными значениями $E_{g}$ твердого раствора InAsSb и энергией фотона в максимуме излучения длинноволновых полос ЭЛ: $\sim 30$ мэВ для гетероструктуры 317 и $\sim 50$ мэВ для гетероструктуры 315 . Кроме того, исследуемые структуры демонстрировали слабую температурную зависимость энергетического положения спектров ЭЛ. Можно сказать, что в диапазоне температур $T=4.2-135 \mathrm{~K}$ она практически отсутствовала. Излучательные переходы с энергией фотона меньше величины $E_{g}$ активной области гетероструктуры, а также слабая температурная зависимость энергии фотона в максимуме регистрируемой полосы ЭЛ позволили сделать предположение о существовании канала интерфейсной излучательной рекомбинации вблизи гетерограницы II типа. Ранее нами было установлено, что гетеропереходы в системе InAs/InAs ${ }_{1-y} \mathrm{Sb}_{y}$ для составов $y<0.13$ являются гетеропереходами I типа, тогда как в системе $\mathrm{InAs}_{1-y} \mathrm{Sb}_{y} / \mathrm{InAs}_{1-x-y} \mathrm{Sb}_{y} \mathrm{P}_{x}$ для составов тройных твердых растворов $y>0.14$ могут формироваться гетерограницы II типа [17].

В гетероструктурах, рассматриваемых в настоящей работе, разрывы энергетических зон на гетерогранице InAsSb/InAsSbP формируют потенциальные барьеры, достаточные для одновременного ограничения движения носителей заряда противоположного знака вблизи интерфейса: дырок в валентной зоне широкозонного барьерного слоя InAsSbP и электронов в зоне проводимости узкозонного активного слоя InAsSb. Благодаря особенностям конструкции гетероструктуры InAs/InAs $\operatorname{In}_{1-y} \mathrm{Sb}_{y} / \mathrm{InAs}_{1-x-y} \mathrm{Sb}_{y} \mathrm{P}_{x}$, за счет большой величины разрывов зон в зоне проводимости $\left(\Delta E_{C}=200\right.$ мэВ) и в валентной зоне $\left(\Delta E_{V}=70\right.$ мэВ $)$ при подаче прямого смещения на гетерогранице InAsSb/InAsSbP II типа образуются потенциальные ямы для электронов и дырок с уровнями размерного квантования, которые пространственно разделены интерфейсом [17]. При достижении определенных концентраций локализованных носителей заряда возникает ненулевая вероятность „непрямой“ (туннельной) излучательной рекомбинации через интерфейс между этими уровнями. В результате локализации носителей заряда на гетерогранице практически отсутствует температурная зависимость спектрального положения максимумов ЭЛ, которое определяется в основном перекрытием зон $\left(E_{S}\right)$ гетероперехода II типа $\operatorname{InAs}_{1-y} \mathrm{Sb}_{y} / \mathrm{InAs}_{1-x-y} \mathrm{Sb}_{y} \mathrm{P}_{x}$. Известно, что увеличение концентрации атомов сурьмы в твердом растворе $\operatorname{InAs}_{1-y} \mathrm{Sb}_{y}$ приводит к уменьшению ширины запрещенной зоны и, как следствие, к уменьшению величины энергетического зазора на гетерогранице II типа. При этом величина $E_{S}$ зазора не зависит напрямую от величины $E_{g}$ тройного твердого раствора $\operatorname{InAs}_{1-y} \mathrm{Sb}_{y}$, вследствие чего для составов, близких друг другу, наблюдается сильное расхождение между расчетной величиной запрещенной зоны InAs $\sin _{1-y} \mathrm{Sb}_{y}$ и энергией фотона интерфейсного перехода на гетерогранице $\operatorname{InAs}_{1-y} \mathrm{Sb}_{y} / \operatorname{InAs}_{0.41} \mathrm{Sb}_{0.28} \mathrm{P}_{0.40}$.
На основании данного предположения можно оценить величины энергетических зазоров в гетеропереходах II типа как: $E_{S}(y=0.15)<h v(317)=0.287$ эВ и $E_{S}(y=0.16)<h v(315)=0.255$ эВ. Последнее значение хорошо согласуется с величиной энергетического зазора гетероперехода II типа $E^{\prime \prime}=0.225$ эВ, полученной в работе [17]. Также в работе [17] была рассмотрена зонная диаграмма гетероструктуры InAs/InAs ${ }_{0.84} \mathrm{Sb}_{0.16} / \mathrm{InAs}_{0.41} \mathrm{Sb}_{0.28} \mathrm{P}_{0.40}, \quad$ иллюстрирующая формирование потенциальных ям для электронов в зоне проводимости и дырок в валентной зоне за счет изгибов зон на гетерогранице $\operatorname{InAs}_{0.84} \mathrm{Sb}_{0.16} / \mathrm{InAs}_{0.41} \mathrm{Sb}_{0.28} \mathrm{P}_{0.40}$ при приложении прямого внешнего смещения.

Таким образом, в исследуемых гетероструктурах в диапазоне температур 4.2-180K преобладали интерфейсные излучательные рекомбинационные переходы между локализованными состояниями электронов и дырок на гетерогранице II типа, позволившие минимизировать влияние температуры на рабочую длину волны излучения светодиода. С повышением температуры до $300 \mathrm{~K}$ вследствие ослабления локализации носителей на интерфейсе вклад данных переходов падал, и начинали доминировать переходы, вызванные рекомбинацией носителей в объеме активного слоя InAsSb.

\section{4. Заключение}

В работе представлены результаты исследования электролюминесценции асимметричных светодиодных гетероструктур InAs/InAs ${ }_{1-y} \mathrm{Sb}_{y} / \mathrm{InAsSbP}$ с мольной долей $\mathrm{InSb}$ в активной области $y=0.15$ и $y=0.16$ в диапазоне температур $4.2-300 \mathrm{~K}$. Показано существенное изменение спектральных характеристик при повышении температуры и увеличении содержания InSb в тройном твердом растворе InAsSb активного слоя. Определено формирование ступенчатого гетероперехода II типа на гетерогранице $\mathrm{InAs}_{1-y} \mathrm{Sb}_{y} / \mathrm{InAs}_{1-x-y} \mathrm{Sb}_{y} \mathrm{P}_{x}$ для исследованных составов. Показано, что преобладание интерфейсных излучательных переходов на гетерогранице InAsSb/InAsSbP в узкозонной гетероструктуре InAs/InAsSb/InAsSbP позволяет подавить температурный сдвиг длины волны излучения светодиода при увеличении температуры вплоть до $T=180 \mathrm{~K}$.

\section{Конфликт интересов}

Авторы заявляют, что у них нет конфликта интересов.

\section{Список литературы}

[1] D. Ting, A. Soibel, A. Khoshakhlagh, S. Keo, B. Rafol, A. Fisher, B. Pepper, E. Luong, C. Hill, S. Guhapala. Infr. Phys. Technol., 97, 210 (2019).

[2] D. Jung, S. Bank, M.L. Lee, D. Wasserman. J. Opt., 19, 123001 (2017).

[3] S.D. Smith, J.G. Crowder, H.R. Hardaway. Proc. SPIE, 4651 (1), 157 (2002). 
[4] М. Айдаралиев, Н.В. Зотова, С.А. Карандашев, Б.А. Матвеев, М.А. Ременный, Н.М. Стусь, Г.Н. Талалакин. ФТП, 35, 619 (2001).

[5] A. Krier, H.H. Gao, V.V. Sherstnev, Y. Yakovlev. J. Phys. D: Appl. Phys., 32, 3117 (1999).

[6] S.P. Svensson, W.L. Sarney, D. Donetsky, G. Kipshidze, Y. Lin, L. Shterengas, Y. Xu, G. Belenky. J. Appl. Opt., 56, B58 (2017).

[7] В.В. Романов, М.В. Байдакова, К.Д. Моисеев. ФТП, 48, 753 (2014).

[8] А.С. Головин, А.А. Петухов, С.С. Кижаев, Ю.П. Яковлев. Письма ЖТФ, 37 (11), 15 (2011).

[9] A.A. Semakova, V.V. Romanov, K.D. Moiseev, N.L. Bazhenov, K.D. Mynbaev. J. Phys. Conf. Ser., 1482, 012023 (2020).

[10] В.В. Романов, Э.В. Иванов, К.Д. Моисеев. ФТТ, 61, 1699 (2019).

[11] М.М. Григорьев, Э.В. Иванов, К.Д. Моисеев. ФТП, 45, 1386 (2011).

[12] Y.P. Varshni. Physica, 4, 149 (1967).

[13] Z.M. Fang, K.Y. Ma, D.H. Jaw, R.M. Cohen, G.B. Stringfellow. J. Appl. Phys., 67, 7034 (1990).

[14] Y. Lacroix, C.A. Tran, S.P. Watkins, M.L.V. Thewalt. J. Appl. Phys., 80, 6416 (1996).

[15] J.A. Van Vechten, T.K. Bergstresser. Phys. Rev. B, 1, 3351 (1970).

[16] Landolt-Börnstein. Handbook, Numerical Data, Ser. III, v. $17 \mathrm{a}$, ed. by O. Madelung (Springer, Berlin, Heidelberg, 1982).

[17] В.В. Романов, Э.В. Иванов, К.Д. Моисеев. ФТТ, 62, 1822 (2020).

Редактор Г.А. Оганесян

\title{
Suppression of wavelength temperature dependence in heterostructures with staggered type II heterojunction InAsSb/InAsSbP
}

\author{
A.A. Semakova ${ }^{1}$, V.V. Romanov ${ }^{2}$, N.L. Bazhenov ${ }^{2}$, \\ K.D. Mynbaev' ${ }^{2}$ K.D. Moiseev ${ }^{2}$ \\ 1 ITMO University, \\ 197101 St. Petersburg, Russia \\ 2 loffe Institute, \\ 194021 St. Petersburg, Russia
}

\begin{abstract}
The results of a study of the electroluminescence of the asymmetric InAs/InAs ${ }_{1-y} \mathrm{Sb}_{y} / \mathrm{InAsSbP}$ LED heterostructures with a molar fraction of InSb in the ternary solid solution in the active region $y=0.15$ and $y=0.16$ in the temperature range $4.2-300 \mathrm{~K}$ are presented. Based on the experimental data, the formation of a staggered type II heterojunction at the $\operatorname{InAs}_{1-y} \mathrm{Sb}_{y} / \mathrm{InAsSbP}$ heterointerface was determined. The dominant contribution of the interface radiative transitions at the type II heterointerface in the temperature range $4.2-180 \mathrm{~K}$ was shown, which makes it possible to minimize the temperature dependence of the operating wavelength of the LEDs.
\end{abstract}

